\title{
Building Cryo-EM at Genentech to Enable Research and Drug Discovery
}

Alberto Estevez $^{1 *}$, Chris Arthur ${ }^{1}$, Lionel Rouge ${ }^{1}$, Marc Kschonsak ${ }^{1}$, Alexis Rohou ${ }^{1}$, and Claudio Ciferri ${ }^{1}$

${ }^{1}$ Department of Structural Biology- Cryo-EM Unit. South San Francisco, California, United States of America.

* Corresponding author: albertoe@gene.com

Cryo-EM at Genentech represents an integral part of the Structural Biology department, complementing $\mathrm{X}$-ray crystallography and NMR and has become crucial for structure determination and rational small molecule drug design. In addition, we support several basic research projects with the goal of understanding the mechanistic details of important biological systems [1-3].

An initial strategic investment in cryo-EM infrastructures in 2015, completed by late 2017, enable our facility to accommodate two microscopes: a Talos 200C screening microscope (ThermoFisher Scientific, Waltham, MA), equipped with 4K Ceta-D CMOS detector and a Titan Krios G2 (ThermoFisher Scientific, Waltham, MA) fitted with K2 summit direct detector equipped with a BioQuantum energy filter (Gatan, Pleasanton, CA). These infrastructures were key in supporting several small molecule pipeline projects with high-resolution structure and medicinal chemistry design. Following this success, a new investment will expand our effort in cryo-EM by incorporating in our facility by 2020 a Glacios microscope (ThermoFisher Scientific, Waltham, MA) fitted with a K2 direct detector (Gatan, Pleasanton, CA) and an additional Titan Krios' equipped with a BioQuantum energy filter and a K3 direct detector (Gatan, Pleasanton, CA).

To date, this investment represents the largest effort in cryo-EM in industry, worldwide. All microscopes are outfitted with automated data acquisition software EPU (ThermoFisher) and Serial EM (Boulder laboratory for 3D electron microscopy). Software is also currently being evaluated for on-the-fly data processing [4]. The increasing amount of analyzed samples and generated data has required the development of a novel Lab Information Management System (LIMS), called gP2S (Genentech Protein 2 Structure), capturing every step in between sample preparation and PDB model generation. Expansion of our facility requires an ongoing training of current staff as well increasing our critical mass.

Along with expanding our instrumentation we have established best practices in sample generation. Choosing the right construct and purification method is addressed early on in project initiation [5]. To enable our iterative workflow we leverage a high throughput small scale expression and purification platform with capabilities of 96 samples in a plate based format for simultaneous automatic purification from E.coli, BEVS and Mammalian cells ( $>10,000$ proteins/year). This purification method is highly sensitive $(0.1 \mathrm{mg} / \mathrm{L})$ and uses a variety of purifications tags like His, GST, MBP, FLAG, Fc, Strep, and Sumo. Purified proteins can be intracellular, secreted, membrane bound, or multi protein complexes. This system is also designed to handle detergent screening which is particularly useful with membrane proteins. Results obtained by this method can be scaled up to provide large amounts of protein sample.

In addition, grid preparation and ice thickness optimization has been carefully investigated. Physics of electron optics teach us that in order to transfer high-resolution information from the sample to the image, its thickness needs to be between $20-40 \mathrm{~nm}$. In fact, imaging samples thicker than $40 \mathrm{~nm}$ dampens highresolution signals with no transferred information past $4 \AA$. Commercially available cryo-EM grids often 
lead to sample thickness of $>100 \mathrm{~nm}$, limiting the achievable resolution. To address this issue, we worked with grid manufacturers to improve grid manufacturing for consistency of optimal ice thickness. These improved grids were able to deliver reproducible, thin samples for imaging and consequently higher resolution data. We have also established a matrix screen to improve sample deposition onto grids for addressing preferential orientation and optimize freezing [6]. This matrix can be screened on our screening microscope and the best conditions put in queue for high resolution data collection on our titan Krios. New instruments are also being designed to automatically deposit samples onto grids for immediate vitrification eliminating the blotting procedure $[7,8]$. These tools, currently under development, will likely provide reproducible and consistent freezing conditions and are currently under investigation by our group.

We are currently increasing our interest in using cryo-EM to structurally enable smaller proteins complexes. In addition to published strategies for determining the structure of protein complexes smaller than $100 \mathrm{kD}$ [9], we make use of fabs and multi fab formats to structurally enable small protein complexes or protein domains. To this end, we take advantage of our in-house expertise in antibody generation and optimization of high affinity antibody fragments.

Micro ED is also a workflow that is under investigation. The expansion of our facility is designed to explore the potential of this technique as a transformative workflow toward small molecule drug design, macrocycles and protein structure determination.

At Genentech we are fortunate to have tremendous amounts of expertise in the same location and determined in succeeding toward a common goal. Our approach is aimed towards lowering turnaround time and accelerating project progression. To support this approach, it is vital that we implement the correct workflows and tools to enable the collection of high-quality data.

\section{References}

[1] Nadia Martinez-Martin, et al., Cell 174 (2018), p.1158.

[2] Hui Xu, et al., Cell volume 174 (2019), p.702.

[3] Clairfeuille, et al., Science, Published online, DOI:10.1126/science.aav8573 (2019)

[4]. www.warpem.com (February 20, 2019)

[5] Estevez et al., M\&M 2018

[6] Christopher Arthur et al., in preparation

[7] www.cryosol-world.com/vitrojet (February 20, 2019)

[8] Venkata P. Dandey, et al., Journal of Structural Biology 202 (2018), p.161.

[9] Mark A. Herzik, et al., bioRxiv (2018) http://dx.doi.org/10.1101/489898. (December 7, 2018) 